\title{
15 Teacher training as a key issue for the integration of computer technologies
}

\author{
Michèle Artigue \\ Equipe DIDIREM \\ Université Paris 7 \& IUFM de Reims \\ France
}

\begin{abstract}
In spite of many institutional actions, and the enthusiasm and militancy of pioneers, the integration of computer technologies into secondary mathematics teaching develops very slowly in France. In this text we point out some obstacles to integration which seem specially resistant-the limited 'educational legitimacy' of computer technologies, the underestimation of computer transpositive processes, the opposition between the technical and conceptual dimensions of mathematical activity, the relationship to instrumentation - and defend the thesis that the poor sensitivity of the usual teacher training to these obstacles partially explains its poor efficiency.
\end{abstract}

\section{Keywords}

Integration, symbolic manipulation systems, teacher education.

\section{INTRODUCTION}

Everywhere in the world, in spite of incentive actions and institutional support, in spite of the increasing role played by computers in our social and cultural life, the integration of computer technologies into mathematics secondary teaching does not develop very fast. France does not escape this general situation.

Since 1980, the 'rational' use of pocket calculators is an explicit aim of the secondary mathematics curriculum and all types of calculators can be used in national and regional examinations. Nevertheless, even today, only a small minority of teachers (about $15 \%$ ) takes this aim in charge, seriously. The great 
majority tolerates calculators but leaves their instrumentation and use to the 'private mathematical life' of students. The negative effects of such a situation, relative to the teaching and learning of the concept of limit, are well documented in the research of Trouche, (1996).

For more than ten years the Ministry of Education has supported and developed a lot of actions in order to impulse the integration of new technologies into teaching: mixed licences which allow schools to buy selected software at reduced cost; financial support of innovative and research groups who take charge of the production of specific software and didactic material, and whose production is widely dispersed by the Ministry; training sessions for teachers taking place during the academic time, summer schools, etc. Nevertheless, even in the many schools now provided with good quality equipment, the use of computers for mathematics teaching remains marginal.

The poor return from all these actions and the resistance of the educational system, in spite of the enthusiastic militancy of pioneers, naturally leads to question the principles and strategies which govern teacher training and their adequacy to the current situation. In this paper, we analyse, in didactical terms, the situation in France. More precisely, we point out some obstacles to integration which, in our opinion, are not taken seriously enough into account in teacher training, and hence contribute to its limited efficiency.

\section{OBSTACLES TO INTEGRATION}

\section{The 'educational legitimacy' of computer technologies}

The resistance of the educational system to computer technologies cannot be analysed without taking into account more general issues, such as the legitimacy of teaching means. In order to tackle this question, we find it useful to distinguish different dimensions for legitimacy, at least the social-cultural, epistemologicalscientific and institutional-educational dimensions. There is no doubt that computer technologies are strongly legitimate as far as the first dimensions are concerned, but this is not enough to ensure their educational legitimacy. Last year, in France, this fact was evidenced by the passionate discussions generated by the commercialisation of the TI-92.

Cultural and scientific integration of computer technologies gives evident arguments in favour of their educational legitimacy: briefly speaking, educational systems cannot reject computer technologies without losing social credibility. But, in order to motivate teachers to pay the price of change, such external arguments are not sufficient. Teachers want to be convinced that the internal efficiency of the educational system will be increased by such a change in teaching means. Computer technologies are thus asked to prove that they can help teachers to face the recurrent difficulties of mathematics teaching and learning and also, for instance, help them to face the new difficulties arising from social crisis. 
In fact, the insufficient pressure of cultural and scientific legitimacy generates a kind of vicious circle: in order to overcome persistent resistance, promoters of change are more or less consciously induced to minimise the costs and difficulties of integration and to overestimate its potential benefits for mathematics teaching and learning. Such strategies are helpful in the short term as they allow innovative practices to survive and even locally develop in bad ecological conditions. In the longer term, they tend to create obstacles to the integration they want to support as they tend to obscure some major difficulties teachers will have to face and, as a consequence, do not promote the development of efficient adaptive means. Integration remains thus trapped in a transitory phase of 'pioneer militancy', instead of reaching a maturity phase.

In our opinion, teacher training is far from having escaped this vicious circle and this fact contributes to explain its limited efficiency.

\section{The underestimation of issues linked to the computer transposition of mathematical knowledge}

The theory of didactic transposition was introduced by Chevallard (1985) in the early eighties in order to better take into account the complexity of processes which govern the institutional life of teaching contents and to provide researchers with operational tools for such an ecological study. Computer technologies increase the complexity of these transpositive processes by adding a new element: the 'computerisation' of mathematical knowledge.

Didactical research is more and more sensitive to these new transpositive processes and their possible didactic effects (Balacheff, 1994) but teacher training programs do not pay so much attention to them. One can see there is, at least partially, some negative effect of the legitimacy problems mentioned above: by devoting attention to the existing discrepancies between school mathematical knowledge and its computerised versions, one risks to weaken their educational legitimacy. This is specially the case for Computer Algebra Systems (CAS) software, more recently introduced in secondary teaching and thus more fragile.

Research carried out about DERIVE, both with desk top computers and the TI92, obviously shows the dangers of such an underestimation (Abboud et al., 1995). Observations of DERIVE classroom sessions, when compared with teachers' expectations, put to the fore:

- the poor awareness of discrepancies teachers and researchers spontaneously have, when preparing or simulating a session (as they unconsciously compensate for them);

- the important 'perturbations', regularly produced by slight differences, if they are not quickly identified and properly managed;

- the spontaneous tendency to consider the didactic effects of such differences as parasite phenomenas which have to be avoided by a careful choice of situation variables, in a posteriori analysis (that is to say, to negate the fact that, 
resulting from computer transpositive processes, they are in some sense unavoidable);

- the learning potential they have if one overcomes this spontaneous tendency and becomes aware of the possibilities resulting from the comparison of two slightly different transpositive worlds.

For instance, in the experimental work developed with beginners in algebra, this comparison allowed the identification of criteria used by students to interpret and work out algebraic and pre-algebraic expressions, which remained invisible in the standard paper and pencil environment, as well as to introduce fruitful discussions about the distinction between mathematical rules and conventions. In the same way, on-going research with the TI-92 shows that the peculiarities of the automatic evaluation of expressions in exact mode can motivate efficient reflexive work about the 'sense' and 'denotation' of algebraic expressions (Delgoulet and Guin, 1997).

\section{The opposition between the technical and conceptual dimensions of mathematical activity}

From a cognitive point of view, the traditional discourse about computer technologies is grounded in the opposition between the technical and conceptual dimensions of mathematical activity, this opposition going along with an underestimation of the technical dimension. Computer technologies are presented as inducing reflective, strategic and conceptual functioning by freeing the student from technique. This vision is not independent from the privileged role these technologies have been given for renewing teaching practices perceived as too narrowly technical and making them more compatible with the dominant constructivist learning theories. Anthropological and cultural approaches are challenging this schematic vision now (Noss and Hoyles, 1996; Chevallard, 1991), but at least in France, the world of teacher training remains under its strict influence.

The detailed observations we made during the last five years with DERIVE and the TI-92 showed the inadequacy of such an idyllic vision (Artigue, 1997). In these environments, due to specific adaptive processes and their economy, two opposite tendencies are at play:

- the first one effectively favouring the reflexive and strategic work teachers would like to induce;

- the second one, conversely, tending to save reflection, favour blind action or atomise the solving process into a great number of micro-actions without evident coherence.

Only a careful management of the didactic variables of situations enables the establishment of an adequate equilibrium between these tendencies. Moreover, except if students are performing routinised tasks, relationships between the technical and conceptual dimensions of mathematical activity appear to be 
strongly dialectic and mutually dependent: it does not really make sense to set them in opposition.

Once more, it seems that most teacher training programs do not give teachers the means really to overcome this dichotomy and seriously investigate (a) how the equilibrium between the two dimensions can be altered by the integration of computer technologies, and (b) what criteria can be used in order to understand, beyond mere action, the kind of mathematical activity students engage in during computer sessions and the situation variables which allow to control and improve this mathematical activity.

\section{Relationship to 'instrumentation'}

Mathematics teaching has been used to living in environments poor in technology. Integration of computers introduces a radical change and obliges us to take into account issues related to the transformation of material artefacts into mathematical instruments (Rabardel, 1995):

- Which learning processes underlie such a transformation and how to manage them?

- What relationships with mathematical knowledge are aimed at by the institution?

- How to deal with eventual discrepancies and conflicts?

Answers to such questions are far from being obvious. Once more, when reflecting on them, one has to face obstacles linked to cultural and ideological dominant positions. If mathematical legitimacy is solely attached to the usual instruments of mathematical activity, how does one justify the price to pay in order to transform computers into mathematical instruments ?

In teacher training, this inevitably leads to stress the conviviality and immediate accessibility of software and calculators, and conversely to underestimate the work necessary in order to instrument them. Research results clearly show the limitations of such an approach. Let us give one example, referring once more to on-going research with the TI-92 (Artigue et al., 1997). Experiments carried out with scientific students at high school level tend to prove that, for these students who are used to working with graphic calculators, familiarity is quickly obtained. But, familiarity is only a first level of instrumentation. Briefly speaking, students are able to use the machine efficiently if it directly gives the desired answer. Faced with an algebraic expression of a derivative which is not in the form given in the text or obtained by paper and pencil means, they are not so obviously able to use the machine to pilot the necessary transformations; they are not necessarily sensitive to graphic phenomenas linked to discretisation even if the results they obtain in the symbolic mode are contradictory with those they have got in the graphic mode; they do not clearly differentiate between approximate and exact results all the more as, even if 
the exact mode is selected, results given in most applications are still approximations.

Further, the TI-92 opens widely the range of possible actions, even nearly equivalent actions, and students are faced with the necessity of selecting and finding a coherent way in this complex world to develop instrumented techniques'. These instrumented techniques, not officially worked and institutionalised the way that standard paper and pencil techniques are, take more time to stabilise and thus more time to become a matter of routine. For a quite long time they remain more sensitive to small perturbations than one might expect.

The decisions the teacher has to take at this level are not without influence:

- What functions and commands have to be introduced in order to benefit from the potential of the machine, without complexifying to much the student's instrumental work?

- How to organise this progressive introduction?

- How to manage the necessary evolution of the didactic contract taking into account what has to be done by hand and what is left to the machine?

- How to simultaneously manage, in classroom activities, the progression in standard mathematical learning and in the instrumentation of the machine; two progressions which are strongly dependent but whose possible relationships are not so well known?

The evolution we have noticed in the teachers' mastery of these problems along these two first years of experimental work with the TI-92 and its positive didactic effects are evidence of the importance of these questions. They have to be seriously addressed in teacher training, but this will be difficult if the legitimacy of the specific apprenticeships required by instrumentation is not firstly recognised.

\section{OBSTACLES AND TEACHER TRAINING STRATEGIES}

Up to now, we have tried to point out obstacles to the integration of computer technologies which, in our opinion, deserve more attention in teacher training than is usually the case. Without pretending to be exhaustive, we chose to focus on obstacles linked to dominant cultural representations. These representations unconsciously shape teachers' expectations with respect to teacher training. They also shape the way teachers interpret and personally integrate the professional experience they have with computers. This makes them specially resistant to change.

Faced with these difficulties, teacher training based on innovative values and militancy has shown evident limits. For the reasons mentioned above, our personal conviction is that such resistant obstacles will not be overcome without giving didactic analysis a more important role in teacher training, and without 
providing teachers with didactic tools allowing them to analyse transpositive processes, to identify the didactic variables of situations and pilot them, and to analyse their professional techniques and the way these are modified by the use of computer technologies.

Abboud (1994) clearly demonstrates that this is not an easy task, even if standard teacher training in this area does not limit itself to technical issues and really tries to address the pedagogical implications of the use of computer technologies. Teachers generally become teacher trainers in this area because they are known to be regular and successful users of computer technologies with their students. They certainly are good experts in the use of computer technologies for mathematics secondary teaching, but as it is normal for experts, most of the competencies they have developed have become routine. They are no longer aware that they had to learn them, they are not necessarily able to analyse them. Moreover they do not necessarily have a strong didactic culture. Most often, the didactical dimension of teacher training is based on 'imitative' strategies: it is organised around situations trainers have experienced with their own students, some nice situations evidencing the interest of computer technologies and their innovative power for mathematics teaching. These are generally worked by using 'homology' techniques, that is to say, by putting teachers in student positions and simulating the classroom life of such a situation. If training sessions are discontinuous, teachers are invited to try some of these situations, possibly with the necessary adaptations, with their own students and, in some further session, time is devoted to reports on these experiments and an analysis of the reports.

We are convinced that these strategies do not enable students to overcome such resistant obstacles as those mentioned above and do not necessarily provide teachers with the didactic tools they need. Efficient teacher training cannot only rely on imitative strategies which cannot correctly take into account the differences between experts and novices, homology techniques obscure the fact that one's mathematical knowledge strongly shapes the use of computer technologies, and if teachers are not provided with didactic tools for analysis, if observations and experiments are not carefully prepared, they only serve to reinforce initial representations or perceptions.

As stressed by Abboud (op. cit.), the didactic investment we ask for requires more efficient links between research and teacher training. This is the reason why, for instance, we decided to use the material collected in our DERIVE research in order to produce didactic resources for teacher training (Lachambre and Abboud, 1996). For this purpose, we selected classroom sessions in the experimental work and organised the corresponding data and analysis as resources for a training module on DERIVE. Each module consists of five parts :

- a synthetic presentation and a priori analysis of the session,

- the material prepared for students; 
128 Information and communications technologies in school mathematics

- a synthetic research analysis of the possible interest of the session for teacher training: identifying interesting points to be worked on at a technical level, at a transpositive level, at a cognitive level, and at a didactic level;

- some selected protocols and data which seem of particular interest in order to support this work with precise data (which, for evident reasons, are out of range of teacher trainers and innovators, if they are not engaged in research projects);

- an indicative strategy for using this module in teacher training sessions.

How much such a material can be really helpful for teacher trainers, remains for us an open question-to be investigated within the framework of a specific research project in the near future.

\section{REFERENCES}

Abboud, M. (1994) L'intégration de l'outil informatique à l'enseignement secondaire des mathématiques: symptômes d'un malaise, Doctoral thesis, Université Paris 7.

Abboud, M., Artigue, M., Drouhard, J. P. and Lagrange, J. B. (1995) Une recherche sur le logiciel DERIVE, Cahier de DIDIREM, 3 (special issue), IREM, Paris 7.

Artigue, M. (1997) Computer environments and learning theories in mathematics education, in B. Barzel (ed.), Teaching Mathematics with DERIVE and the TI92, Proceedings of the International DERIVE and TI-92 Conference, Bonn, $1-17$.

Artigue, M., Defouad, B., Dupérier, M., Juge, G. and Lagrange, J. B. (1997) L'intégration de calculatrices complexes à l'enseignement scientifique au lycée. Research Report, Equipe DIDIREM, IREM, Paris 7.

Balacheff, N. (1994) Didactique et intelligence artificielle. Recherches en Didactique des Mathématiques, 14(1-2), 9-42.

Chevallard, Y. (1985) La transposition didactique. La Pensée Sauvage, Grenoble

Chevallard, Y. (1991) Dimension instrumentale, dimension sémiotique de l'activité mathématique, Séminaire de didactique des mathématiques et de l'informatique, LSD-IMAG, Grenoble.

Delgoulet, J. and Guin, D. (1997) Etude des modes d'appropriation de calculatrices graphiques et symboliques dans une classe de seconde, IREM de Montpellier.

Lachambre, B. and Abboud, M. (1996) Training of mathematics teachers. The International DERIVE Journal, 3(3), 109-124.

Noss, R. and Hoyles, C. (1996) Windows on Mathematical Meanings: Learning Cultures and Computers. Kluwer Academic Press.

Rabardel, P. (1995) Les hommes et les technologies-Approche cognitive des instruments contemporains. A.Colin, Paris. 
Trouche, L. (1996). A propos de l'apprentissage de la notion de limite de fonctions dans 'un environnement calculatrice': étude des rapports entre processus de conceptualisation et processus d'instrumentation. Doctoral thesis, Université de Montpellier II.

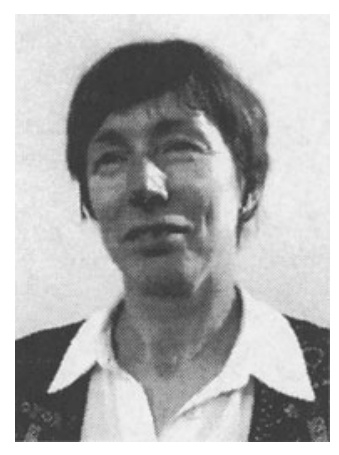

Michèle Artigue is professor at the IUFM of Reims and responsible of the research team DIDIREM in the didactics of mathematics at the University of Paris 7. After a doctoral thesis in mathematical logic, she dedicated to the didactics of mathematics and got an habilitation in this area. Her research work mainly deals with epistemological issues, the didactic of Analysis, at secondary and university level and the use of computer technologies for mathematics teaching. From 1992 to 1995 , she was responsible for a national research project on the use of DERIVE in secondary schools, supported by the Ministry of Education and she is now piloting a research project on the integration of the calculator TI-92 in scientific series at high school level. She is the chief editor of the journal Recherches en Didactique des Mathématiques. 